\title{
Matrix Formulation of EISs of Graphs and Its Application to WSN Covering Problems
}

\author{
Yongyi Yan $\left(\mathbb{D},{ }^{1}\right.$ Jumei Yue $\mathbb{D}^{2}{ }^{2}$ and He Deng ${ }^{1}$ \\ ${ }^{1}$ College of Information Engineering, Henan University of Science and Technology, Luoyang, China \\ ${ }^{2}$ College of Agricultural Equipment Engineering, Henan University of Science and Technology, Luoyang, China
}

Correspondence should be addressed to Jumei Yue; yjm@mail.nankai.edu.cn

Received 2 February 2021; Revised 15 March 2021; Accepted 9 April 2021; Published 22 April 2021

Academic Editor: Elena Guardo

Copyright (c) 2021 Yongyi Yan et al. This is an open access article distributed under the Creative Commons Attribution License, which permits unrestricted use, distribution, and reproduction in any medium, provided the original work is properly cited.

In this paper, the problem of formulating and finding externally independent sets of graphs is considered by using a newly developed STP method, called semitensor product of matrices. By introducing a characteristic value of a vertex subset of a graph and using the algebraic representation of pseudological functions, several necessary and sufficient conditions of matrix form are proposed to express the externally independent sets (EISs), minimum externally independent sets (MEISs), and kernels of graphs. Based on this, the concepts of EIS matrix, MEIS matrix, and kernel matrix are introduced. By these matrices' complete characterization of these three structures of graphs, three algorithms are further designed which can find all these kinds of subsets of graphs mathematically. The results are finally applied to a WSN covering problem to demonstrate the correctness and effectiveness of the proposed results.

\section{Introduction}

A graph is a figure composed of vertices and edges and is usually used to describe a specific relationship between things; vertices are used to represent things, and an edge connecting two vertices is used to represent the relationship between the two things. The intuitive diagrammatic representation of relationships has created widespread use of graphs in modeling systems in physical science and engineering problems; actually any system involving a binary relation can be represented by a graph [1]. Graph theory has experienced explosive growth and has come to play a central role in engineering and computer science curricula [2].

Independent set problem of graphs is a key issue in graph theory and control theory and has been applied to many practical problems in the industrial field such as resource selection, facility location, mobile base station location, and logistics center location. $[3,4]$. The problem has become a hot topic in the field of system and control. Yue and Yan [5] gave a matrix formulation of k-internally independent sets and k-maximum internally independent sets of graphs by proposing several new sufficient and necessary conditions of k-internally independent sets and k-maximum internally independent sets. Based on these conditions, they further designed algebraic algorithms that can find all the $\mathrm{k}$-internally independent sets and k-maximum internally independent sets of a given graph. The approach is different from other methods and may offer a new perspective to understand structures of a graph. With regard to the number of independent sets of a graph, Hua [6] proposed an upper bound of the value for the graphs having $k$ cut-edges. Also, he described corresponding extremal graphs. There are also a series of conjectures on independent sets of graphs. Deng, Guo, and Zang [7] proved Chvatal's conjecture on maximal independent sets of a graph. Later, the conjecture was generalized by Ding [8], which was then proved by Sun and $\mathrm{Hu}$ [9].

In recent years, a new matrix analysis tool, called semitensor product of matrices (STP of matrices), has been developed [10]. The STP of matrices generalizes the traditional product of matrices by allowing any two matrices; the number of columns of the former is not equal to that of rows of the latter to participate in operation; and almost all the major properties of the traditional product hold true for the 
STP of matrices. Theoretically, the STP of matrices can be applied in most of engineering and science fields, especially for the problems that can be presented as a discrete mathematical model. The STP of matrices is now well established and has been found to be very useful in many areas [11-19].

Based on the STP of matrices, a matrix approach to investigate graph problems has been established recently. Wang, Zhang, and Liu [20] introduced the STP of matrices to the domain of graph theory and proposed a matrix approach to investigate serval problems such as the internally stable set problem, absolutely maximum internally stable set problem, maximum weight stable set problem, and vertex coloring problem. Several new results and algorithms were proposed. Afterwards, the matrix approach was applied to many problems in the field of graph theory. In [21], Xu considered the coloring problem of robust graphs and presented several new results by formulating the coloring problem as an optimization problem. With these new conclusions, a new timetabling scheme is developed. Later, $\mathrm{Xu}$ and Wang $[22,23]$ advanced the study to the problems of conflict-free coloring and fuzzy graph coloring. Zhong et al. [24] used the matrix approach to discuss the way of searching minimum stable sets and cores of graphs. Meng, Feng, and Li [25] pushed the method towards the hypergraphs area and investigated transversal and covering problems. In [26], Yue et al. proposed a necessary and sufficient condition of multi-internally stable set of graphs. The presented results are further used to solve the multitrack assignment problem; two solvability conditions are presented.

The advantages of this matrix approach to graph problems are the mathematical formulation and exact decision results for the problems, which are very useful to study other graph problems. The idea of this approach is entirely different from that of existing ones and may be a new perspective to comprehend the structures of graphs in a mode of mathematics.

The motivations of this paper, inspired by the superiorities of the matrix approach to graphs, are to formulate the externally independent sets of graphs in a matrix fashion and to find all the externally independent sets of a graph mathematically. Further, the results are expected to expand to the cases of minimum externally independent sets and kernels of graphs; and the results are used to solve a kind of WSN covering problem and service stations optimal location problem, which serves as a means of examination of the correctness and effectiveness of the proposed results.

The contributions of this paper consist of two aspects. In theory, we established several necessary and sufficient conditions of externally independent sets, minimum externally independent sets, and kernels of graphs. In algorithm, three algorithms were designed which can produce all the externally independent sets, all the minimum externally independent sets, and all the kernels of a graph. These may advance the graph matrix approach in the area of system science.

The paper is organized as follows. In Section 2, we give necessary preliminaries on graphs and the research tool of this paper, as well as the STP of matrices. Section 3 presents the main results of this paper, including theory results and algorithm ones. Section 4 is devoted to discussing differences between the proposed approach and existing methods, as well as the complexity of the proposed algorithms. An application of the results to WSN covering problem, serving as an examination of the correctness and effectiveness of the presented results, is given in Section 5; this is followed by concluding remarks in Section 6.

\section{Preliminaries}

In this section, we give some necessary preliminaries on STP of matrices (semitensor product of matrices) and externally independent sets (EISs) of graphs.

Definition 1 (STP of matrices) (see [10]). For any two matrices $M \in \mathbf{M}_{m \times n}$ and $N \in \mathbf{M}_{p \times q}$, their STP, denoted by $M \ltimes N$, is defined as

$$
M \ltimes N:=\left(M \otimes I_{s / n}\right)\left(N \otimes I_{s / p}\right),
$$

where $s$ is the least common multiple of $n$ and $p$ and $\otimes$ is the Kronecker product.

The following example illustrates the STP of matrices: (1) For two vectors $X=[1,-2,1,-3]$ and $Y=\left[\begin{array}{c}2 \\ -1\end{array}\right]$,
their STP is

$$
X \ltimes Y=[1,-2] \cdot 2+[1,-3] \cdot(-1)=[1,-1] .
$$

(2) For two matrices of dimensions $3 \times 4$ and $2 \times 2, A=\left[\begin{array}{cccc}2 & 2 & 4 & -1 \\ 4 & -1 & 0 & 2 \\ -1 & -2 & 1 & 1\end{array}\right]$ and $B=\left[\begin{array}{cc}-3 & -2 \\ 2 & 1\end{array}\right]$, their STP is

$$
\begin{aligned}
A \ltimes B & =\left[\begin{array}{l}
{[2,2,4,-1] \ltimes\left[\begin{array}{c}
-3 \\
2
\end{array}\right][2,2,4,-1] \ltimes\left[\begin{array}{c}
-2 \\
1
\end{array}\right]} \\
{[4,-1,0,2] \ltimes\left[\begin{array}{c}
-3 \\
2
\end{array}\right][4,-1,0,2] \ltimes\left[\begin{array}{c}
-2 \\
1
\end{array}\right]} \\
{[-1,2,1,1] \ltimes\left[\begin{array}{c}
-3 \\
2
\end{array}\right][-1,2,1,1] \ltimes\left[\begin{array}{c}
-2 \\
1
\end{array}\right]}
\end{array}\right] \\
& =\left[\begin{array}{cccc}
2 & 2 & 0 & 1 \\
-12 & 2 & -8 & 4 \\
5 & -4 & 3 & -3
\end{array}\right] .
\end{aligned}
$$

Remark 1. The STP of matrices is a generalization of the traditional matrix multiplication; when $n=p$, the STP of matrices reduces to the latter. In this paper, the matrix multiplication is the STP of matrices by default, and the symbol $\ltimes$ is also omitted except for special emphasis needed. 
Definition 2 (swap matrix) (see [10]). A swap matrix $W_{[m, n]}$ is an $m n \times m n$ matrix, whose rows and columns are tagged by index $(i, j)$. The rows are organized by the order of multiindex $\operatorname{Id}[j, i ; n, m]$ and the columns by $\operatorname{Id}[i, j ; m, n]$. The element at position $[(I, J),(i, j)]$ is defined as

$$
W_{((I, J),(i, j))}=\delta_{i, j}^{I, J}= \begin{cases}1, & I=i \text { and } J=j, \\ 0, & \text { otherwise. }\end{cases}
$$

Proposition 1 (A property of swap matrix) [10]: For any two column vectors $X \in \mathbf{R}^{m}$ and $Y \in \mathbf{R}^{n}$, the following pseudocommutative law holds:

$$
\begin{aligned}
& W_{[m, n]} \ltimes X \ltimes Y=Y \ltimes X, \\
& W_{[n, m]} \ltimes Y \ltimes X=X \ltimes Y,
\end{aligned}
$$

Definition 3 (dummy operator) (see [27]). The dummy operators $E_{d_{-} \text {latter }}(m, n)$ and $E_{d_{-} \text {former }}(m, n)$ are defined as

$$
\begin{aligned}
E_{d_{-} \text {latter }}(m, n) & =\underbrace{\left[I_{n}, \ldots, I_{n}\right]}_{m}, \\
E_{d_{-} \text {former }}(m, n) & =\underbrace{\left[I_{m}, \ldots, I_{m}\right]}_{n} .
\end{aligned}
$$

For convenience, define $E_{d_{-} \text {latter }}(2,2):=E_{d_{-} \text {latter }}$ and $E_{d_{-} \text {former }}(2,2):=E_{d_{\text {_former }}}$.

Proposition 2 (a property of dummy operator) (see [27]). For any two logical vectors $u \in \Delta_{m}$ and $v \in \Delta_{n}\left(\Delta_{m}\right.$ and $\Delta_{n}$ are described below), the following pseudoelimination laws hold:

$$
\begin{aligned}
E_{d_{-} \text {latter }}(m, n) \ltimes u \ltimes v & =v, \\
E_{d_{-} \text {former }}(m, n) \ltimes W_{[n, m]} \ltimes u \ltimes v & =u .
\end{aligned}
$$

Proposition 3 (negation operator) (see [20]). ie matrix $M_{n}=\delta_{2}[2,1]$ is called the negation operator of 2-valued logical variables (where $\delta_{2}[2,1]$ is given in the following notations). For any 2-valued logical variable $x, M_{n} x=\bar{x}$.

Definition 4 (graph) (see [2]). A graph $G$ is a tuple $G=(V, E)$, where $V$ is a set of objects called vertices and $E$ is a set of 2-element subsets of $V$ called edges.

In a graph $G=(V, E), V=\left\{v_{1}, v_{2}, \ldots, v_{n}\right\}, v_{j}$ is called a neighbor of $v_{i}$ if $\left(v_{j}, v_{i}\right) \in E$; $v_{i}$ is default to be a neighbor of itself. Denote by $N\left(v_{i}\right)=\left\{v_{j} \in V \mid\left(v_{j}, v_{i}\right) \in E\right\}$ the neighbor set of $v_{i}$.

Definition 5 (externally independent set of a graph) (see [20]). A set $S$ of vertices of $G$ is called an externally independent set (EIS) of $G$ if for any a vertex $v_{i} \notin S$ there is a vertex $v_{j} \in S$ such that $v_{j} \in N\left(v_{i}\right)$. An EIS $S$ is called a minimum externally independent set (MEIS) if any proper subset of $S$ is not an EIS. An MEIS with the least number of vertices is called an absolutely minimum externally independent set (AMEIS). The number of vertices of an AMEIS is called the externally independent number of $G$, denoted by $\alpha(G)$.
Definition 6. (unitary adjacency matrix of a graph) (see [27]). For a graph $G$ of $n$ vertices $V=\left\{v_{1}, v_{2}, \ldots, v_{n}\right\}$, the matrix $B=\left[b_{i j}\right]$ is called the adjacency matrix of $G$ :

$$
b_{i j}= \begin{cases}1, & v_{i} \in N\left(v_{j}\right), \\ 0, & v_{i} \notin N\left(v_{j}\right) .\end{cases}
$$

The matrix $A=\left[a_{i j}\right]$ is called the unitary adjacency matrix (UAM) of $G$ :

$$
a_{i j}= \begin{cases}1, & i=j, \\ b_{i j}, & i \neq j .\end{cases}
$$

It is easy to get that

$$
a_{i j}=b_{i j} \vee \delta_{i j}
$$

where $\vee$ denotes the logic product operation, and $\delta_{i j}$ is the Kronecker symbol defined as follows:

$$
\delta_{i j}= \begin{cases}1, & i=j, \\ 0, & i \neq j .\end{cases}
$$

\section{Main Results}

In this section, we present the main results of this paper, including theoretic part and algorithm part. Theoretic results consist of matrix formulations of externally independent set and minimum externally independent set of graphs. Algorithm results contain two algorithms which can find all the externally independent sets and minimum externally independent sets of a graph, respectively. Further, we extend these results to the kernels of graphs by appropriate modification.

\subsection{Matrix Formulation and Algebraic Algorithm of EIS}

Definition 7 (characteristic logical vector of vertex subset) (see [20]). Given a vertex subset $S \subseteq V$ of $G=(V, E)$, the characteristic logical vector of $S$, denoted as $V_{S}=\left[x_{1}, x_{2}, \ldots, x_{n}\right]$, is

$$
x_{i}= \begin{cases}1, & x_{i} \in S, \\ 0, & x_{i} \notin S .\end{cases}
$$

In the framework of the STP of matrices and based on Definition 7 , we define the characteristic value of a vertex subset of a graph. A characteristic value corresponds to a unique vertex subset of a graph.

Definition 8 (characteristic value of vertex subset). For a vertex subset $S \subseteq V$ of $G=(V, E), V_{S}=\left[x_{1}, x_{2}, \ldots, x_{n}\right]$ is its characteristic logical vector. Let $y_{i}=\left[x_{i}, 1-x_{i}\right]^{T}$, $i=1,2, \ldots, n$; the characteristic value of $S$ is defined as

$$
Y_{S}=\ltimes_{i=1}^{n} y_{i}
$$

Remark 2. Since each $y_{i}$ is uniquely determined by $y_{i}=S_{i}^{n} \ltimes Y_{S}$, where $S_{i}^{n}$ is defined in (30), and each set of $y_{i}$ 
uniquely determines $Y_{S}$, the characteristic value $Y_{S}$ of $S$ is uniquely determined by the characteristic logical vector $V_{S}$, and vice versa. Therefore, once one of $Y_{S}$ and $V_{S}$ is known, the other one can then be determined; consequently, the vertex subset $S$ is known.

Theorem 1. Given a graph $G=(V, E)$ with $A=\left[a_{i j}\right]$ being its UAM, $V=\left\{v_{1}, v_{2}, \ldots, v_{n}\right\}$. $G$ contains an externally independent set if and only if there is $1 \leq j \leq 2^{n}$ satisfying

$$
\operatorname{col}_{j}(M)=1_{n},
$$

where

$$
\begin{aligned}
M & =\left[\begin{array}{c}
M_{1} \\
M_{2} \\
\vdots \\
M_{n}
\end{array}\right], \\
M_{i} & =J \ltimes \sum_{i=1}^{n}\left(a_{i j} Q_{j}\right), \quad i=1,2, \ldots, n, \\
J & =[1,0], \\
Q_{j} & =\left(E_{d_{-} \text {latter }}\right)^{n-j} \ltimes W_{\left[2,2^{n-j}\right]} \ltimes\left(E_{d_{-} \text {latter }}\right)^{j-1} .
\end{aligned}
$$

Proof. Necessity: assume that $S$ is an externally independent set of $G$. Let $V_{S}=\left[x_{1}, x_{2}, \ldots, x_{n}\right]$ be the characteristic logical vector of $S$. It follows from Definition 5 that, for a vertex $v_{i}$ not in $S$, corresponding to $x_{i}=0$, there is $j(1 \leq j \leq n)$ such that

$$
\begin{aligned}
& b_{i j}=1, \\
& x_{j}=1 .
\end{aligned}
$$

Equation (16) is equivalent to

$$
x_{i} \vee\left(\bigcup_{j=1}^{n} b_{i j} x_{j}\right)=1 .
$$

From (8) and (17), we have the following:

$$
\bigvee_{j=1}^{n} a_{i j} x_{j}=1
$$

Since both $a_{i j}$ and $x_{j}$ only take the values of 0 and 1, (18) can be further expressed as

$$
\sum_{j=1}^{n} a_{i j} x_{j}=1
$$

On the other hand, set $y_{j}=\left[x_{j}, 1-x_{j}\right]^{T}, j=1,2, \ldots, n$. From (7), we have that

$$
\begin{aligned}
y_{j} & =\left(E_{d \_ \text {latter }}\right)^{n-j} \ltimes y_{j+1} \ltimes y_{j+2} \ltimes \cdots \ltimes y_{n} \ltimes y_{j} \\
& =\left(E_{d \_l \text { latter }}\right)^{n-j} \ltimes W_{\left[2,2^{n-j}\right]} \ltimes y_{j} \ltimes y_{j+1} \ltimes \cdots \ltimes y_{n} \\
& =\left(E_{d \_l \text { latter }}\right)^{n-j} \ltimes W_{\left[2,2^{n-j}\right]} \ltimes\left(E_{d \_l \text { latter }}\right)^{j-1} \ltimes y_{1} \ltimes \cdots \ltimes y_{n} \\
& :=Q_{j} \ltimes Y_{S} .
\end{aligned}
$$

where $Q_{j}=\left(E_{d_{-} \text {latter }}\right)^{n-j} \ltimes W_{\left[2,2^{n-j}\right]} \ltimes\left(E_{d_{-} \text {latter }}\right)^{j-1}$.

Clearly,

$$
\begin{aligned}
x_{j} & =J \ltimes y_{j}, \\
a_{i j} x_{j} & =a_{i j} J \ltimes y_{j}=a_{i j} J \ltimes Q_{j} \ltimes Y_{S} .
\end{aligned}
$$

We thus get from (19) that

$$
\sum_{j=1}^{n}\left(a_{i j} x_{j}\right)=J \ltimes \sum_{j=1}^{n}\left(a_{i j} Q_{j}\right) \ltimes Y_{S}:=M_{i} \ltimes Y_{S} .
$$

where $M_{i}=J \ltimes \sum_{j=1}^{n}\left(a_{i j} Q_{j}\right)$.

Thus, the following equations are equivalent to each other for $i \in\{1,2, \ldots, n\}$ :

(1) $\sum_{j=1}^{n} a_{i j} x_{j}=1$

(2) $M_{i} \ltimes Y_{S}=1$

(3) There is a column of $M_{i}$ which is $1_{n}$

(4) There exists $1 \leq j \leq 2^{n}$ such that $\operatorname{col}_{j}\left(M_{i}\right)=1_{n}$

Note that (19) amounts to

$$
\left\{\sum_{j=1}^{n} a_{1 j} x_{j}=1, \sum_{j=1}^{n} a_{2 j} x_{j}=1, \vdots \sum_{j=1}^{n} a_{n j} x_{j}=1 .\right.
$$

It is easy to know that equation (23) has a solution $\left[x_{1}, x_{2}, \ldots, x_{n}\right]$ if and only if there is $1 \leq j \leq 2^{n}$ such that

$$
\operatorname{col}_{j}(M)=1_{n}
$$

where

$$
M=\left[\begin{array}{c}
M_{1} \\
M_{2} \\
\vdots \\
M_{n}
\end{array}\right] .
$$

The equivalence between (19) and (23) shows the necessity.

(Sufficiency). The sufficiency follows the reversibility from equations (16) to (23).

Remark 3. Theorem 1 describes the condition that a graph contains an externally independent set, based on which we can develop a criterion of judging whether a given vertex subset of a graph is an externally independent set or not.

Theorem 2 Let $A=\left[a_{i j}\right]$ be the UAM of graph $G=(V, E)$. Given a vertex subset $S \subseteq V$ with $V_{S}=\left[x_{1}, x_{2}, \ldots, x_{n}\right]$ being its characteristic logical vector and $Y_{S}=\ltimes_{i=1}^{n} y_{i}=\delta_{2^{n}}^{k}$ being its characteristic value, where $y_{i}=\left[x_{i}, 1-x_{i}\right]^{T}, S$ is an externally independent set of $G$ if and only if

$$
\operatorname{col}_{k}(M)=1_{n} .
$$


where

$$
\begin{aligned}
M & =\left[\begin{array}{c}
M_{1} \\
M_{2} \\
\vdots \\
M_{n}
\end{array}\right], \\
M_{i} & =J \ltimes \sum_{i=1}^{n}\left(a_{i j} Q_{j}\right), \quad i=1,2, \ldots, n, \\
J & =[1,0], \\
Q_{j} & =\left(E_{d_{-} \text {latter }}\right)^{n-j} \ltimes W_{\left[2,2^{n-j}\right]} \ltimes\left(E_{d_{-} \text {latter }}\right)^{j-1} .
\end{aligned}
$$

Proof. Comparing (26) with (14), we know that it is sufficient to show that the $k$-th column of $M$ is equal to $1_{n}$; that is, the $k$-th column of $M_{i}, i=1,2, \ldots, n$ is equal to 1 .

The proof of Theorem 1 shows that $M_{i} \ltimes Y_{S}=1$ if and only if "there is $1 \leq j \leq 2^{n}$ satisfying $\operatorname{col}_{j}\left(M_{i}\right)=1$." Thus, there is only a need to prove that $k$ meets the requirement. Actually, the sizes of $M_{i}$ and $Y_{S}$ are $1 \times 2^{n}$ and $2^{n} \times 1$, respectively. In this situation, the semitensor product of matrices amounts to the traditional matrix multiplication. Thus, $M_{i} \ltimes Y_{S}$ is exactly the $k$-th column of $M_{i}$. It is natural that $M_{i} \ltimes Y_{S}=1$ is equivalent to $\operatorname{col}_{k}\left(M_{i}\right)=1$. Theorem 2 is then proved.

Remark 4. By Theorems 1 and 2, we know that matrix $M$ of (14) or (26) provides the information of all the externally independent sets of a graph. In view of this, we introduce the following definition:

Definition 9 (externally independent set matrix). Matrix $M$ of (14) or (26) in Theorem 1 or 2 is called the externally independent set matrix of graph $G$.

According to the proof of Theorem 1, we can easily get the following conclusion which describes a condition under which a graph has an externally independent set. The difference with Theorem 1 is the usage of a different characteristic logical vector, as Corollary 1 shows.

Corollary 1. Consider graph $G=(V, E)$ whose $U A M$ is $A=\left[a_{i j}\right]$. Give $v_{i} \in V$ a two-valued variable $x_{i} \in D=\{0,1\}$ and let $y_{i}=\left[x_{i}, 1-x_{i}\right]^{T}$. There are externally independent sets in $G$ equivalent to the fact that the following equation has at least one solution:

$$
M \underset{i=1}{\stackrel{n}{\ltimes}} y_{i}=1_{n}
$$

In fact, the number of externally independent sets of $G$ equals that of the solutions. More specifically, an externally independent set is determined by each solution of (28). Further, according to Theorem 2, the number of externally independent sets is equal to that of $1_{n}$-columns of $M$.

The proofs of Theorems 1 and 2, as well as Corollary 1, imply a way to design an algorithm to obtain all the externally independent sets of a graph.
Algorithm 1. For a given graph $G=(V, E)$, let $A=\left[a_{i j}\right]$ be the UAM of $G$, and assign each $v_{i} \in V$ a two-valued variable $x_{i} \in D=\{0,1\}$. Set $y_{i}=\left[x_{i}, 1-x_{i}\right]^{T}$. The following five-step procedure yields all the externally independent sets and minimum externally independent sets of $G$.

Step 1: compute the externally independent set matrix $M$ of graph $G$.

Step 2: check whether $M$ has a column being $1_{n}$. If not, $G$ has no externally independent set and the algorithm ends here. Otherwise, define

$$
K=\left\{l \mid \operatorname{col}_{l}(M)=1_{n}\right\}
$$

Step 3: solve the equation $\ltimes_{i=1}^{n} y_{i}=\delta_{2^{n}}^{l}$ with respect to $y_{i}$ for each $l$ in $K$ by computing $y_{i}=S_{i}^{n} \ltimes \delta_{2^{n}}^{l}$, $i=1,2, \ldots, n$, where

$$
\left\{\begin{array}{l}
\left.S_{1}^{n}=\delta_{2}[\underbrace{1, \ldots, 1}_{2^{n-1}} \underbrace{2, \ldots, 2}_{2^{n-1}}]\right] \\
S_{2}^{n}=\delta_{2}[\underbrace{1, \ldots, 1}_{2^{n-2}} \underbrace{2, \ldots, 2}_{2^{n-2}} \underbrace{1, \ldots, 1}_{2^{n-2}} \underbrace{2, \ldots, 2}_{2^{n-2}}], \\
\vdots \\
S_{n}^{n}=\delta_{2} \underbrace{[\underbrace{12}_{2}, \ldots, \underbrace{12}_{2}]}_{2^{n-1}} .
\end{array}\right.
$$

Step 4: select $y_{i}=\delta_{2}^{1}$ and construct $S_{l}=\left\{v_{i} \mid y_{i}=\delta_{2}^{1}\right\} . S_{l}$ is an externally independent set of $G$. All the externally independent sets of $G$ are

$$
\left\{S_{l} \mid l \in K\right\}
$$

Step 5: the externally independent number of $G$ is

$$
\alpha(G)=\min _{l \in K}\left\{\left|S_{l}\right|\right\},
$$

where $\left|S_{l}\right|$ is the cardinality of $S_{l}$. All the minimum externally independent sets of $G$ are

$$
\zeta=\left\{S_{l}|| S_{l} \mid=\alpha(G)\right\} .
$$

Remark 5. Using Algorithm 1, we can obtain all the externally independent sets of a graph, including the externally independent sets with special properties; for example, each vertex outside an externally independent set is linked with the externally independent set only once, exactly $k$ times, and so forth. In brief, Algorithm 1 offers a way to see the whole picture of the structure of a graph in terms of externally independent sets by producing all of them. 
3.2. Matrix Formulation and Algebraic Algorithm of MEIS Algorithm 1's ability of finding all the externally independent sets of a graph enables us to obtain the minimum externally independent sets of a given graph. In this subsection, we address the algebraic formulation and algorithm of minimum externally independent sets of graphs.

Theorem 3. Consider a graph $G=(V, E)$ with the UAM $A=\left[a_{i j}\right]$. For a given vertex subset $S \subseteq V$ with characteristic logical vector $V_{S}=\left[x_{1}, x_{2}, \ldots, x_{n}\right]$ and characteristic value $Y_{S}=\ltimes_{i=1}^{n} y_{i}=\delta_{2^{n}}^{k}$, where $y_{i}=\left[x_{i}, 1-x_{i}\right]^{T}, S$ is a minimum externally independent set of $G$ if and only if

$$
\operatorname{col}_{k}(M)=0_{n} .
$$

where

(1) $M=J \ltimes Q_{i}-n^{2} J^{n-1} M_{n} E_{d_{-} l a t t e r} \sum_{j=1}^{n}\left(\bar{a}_{i j}\right)$

(2) $J=[1,0] ; Q_{i}=\left(E_{d_{-} \text {latter }}\right)^{n-i} \times W_{\left[2,2^{n-i}\right]} \ltimes\left(E_{d_{\text {_latter }}}\right)^{i-1}$

$M_{n}$ is given in Proposition 3; $\bar{a}_{i j}=1-a_{i j}$.

Proof. (Necessity) Let $V_{S}=\left[x_{1}, x_{2}, \ldots, x_{n}\right]$ be the characteristic logical vector of the minimum externally independent set $S$.

(i) For $x_{i}=0$, we know according to (19) that

$$
\sum_{\substack{j=1 \\ j \neq i}}^{n} a_{i j} x_{j}=1 .
$$

(ii) For $x_{i}=1$, we next prove that

$$
\sum_{\substack{j=1 \\ j \neq i}}^{n} a_{i j} x_{j}=0 .
$$

If this not true, that is, $\sum_{j=1}^{n} a_{i j} x_{j}=1$, it follows then $j \neq i$

from Theorem 1 that the vertex subset with characteristic logical vector $V_{S}=\left[x_{1}, \ldots, x_{i-1}, 0, x_{i+1}, \ldots, x_{n}\right]$ is also an externally independent set; that is, $S^{\prime}=\left(v_{1}, \ldots, v_{i-1}, v_{i+1}\right.$, $\left.\ldots, v_{n}\right)$ is an externally independent set of $G$. This contradicts the fact that $S=\left(v_{1}, \ldots, v_{i}, \ldots, v_{n}\right)$ is a minimum externally independent set of $G$. Thus, (36) holds.

Equations (35) and (36) imply that

$$
x_{i}=\sum_{j=1}^{n}\left(\bar{a}_{i j} \underset{\substack{j=1 \\ j \neq i}}{n} \bar{x}_{j}\right) .
$$

where $\bar{a}_{i j}=1-a_{i j}$.

On the other hand,

$$
\begin{aligned}
& \sum_{j=1}^{n}\left(\bar{a}_{i j} \bigwedge_{\substack{j=1 \\
j \neq i}}^{n} \bar{y}_{j}\right)=\sum_{j=1}^{n}\left(\bar{a}_{i j} \bigwedge_{\substack{j=1 \\
j \neq i}}^{n}\left(M_{n} y_{j}\right)\right) \\
& =\sum_{j=1}^{n}\left(\bar{a}_{i j} M_{n} \bigwedge_{\substack{j=1 \\
j \neq i}}^{n} y_{j}\right)=n M_{n} \sum_{j=1}^{n}\left(\bar{a}_{i j} \bigwedge_{\substack{j=1 \\
j \neq i}}^{n} y_{j}\right) \\
& =n M_{n} \sum_{j=1}^{n}\left(\bar{a}_{i j} E_{d_{-} \text {latter }} y_{i} \bigwedge_{\substack{j=1 \\
j \neq i}}^{n} y_{j}\right) \\
& =n M_{n} \sum_{j=1}^{n}\left(\bar{a}_{i j} E_{d_{-} \text {latter }} \bigwedge_{j=1}^{n} y_{j}\right)=n M_{n} \sum_{j=1}^{n}\left(\bar{a}_{i j} E_{d_{-} \text {latter }} Y_{s}\right) \\
& =n^{2} M_{n} E_{d_{-} \text {latter }} \sum_{j=1}^{n}\left(\bar{a}_{i j}\right) Y_{s} .
\end{aligned}
$$

By (20), we get

$$
x_{i}=J y_{i}=J\left(E_{d \_ \text {latter }}\right)^{n-j} W_{\left[2,2^{n-j}\right]}\left(E_{d \_l \text { latter }}\right)^{j-1} Y_{S}:=J Q_{i} Y_{S} .
$$

Substitution of $x_{j}=J y_{j}$ and equations (38) and (39) into (37) gives the necessity.

Sufficiency: suppose that (34) holds; it follows from the reversibility of equation (37) to (39) that the characteristic logical vector of $S, V_{S}=\left[x_{1}, x_{2}, \ldots, x_{n}\right]$, satisfies (35) and (36).

If $x_{1}=x_{2}=\cdots=x_{n}=1$, then $\sum_{j=1}^{n} a_{i j} x_{j} \geq \sum_{j=1}^{n} a_{j j}=1$; therefore, $S$ is an externally independent set of $G$. If there is $x_{i}=0$, then

$$
\sum_{j=1}^{n} a_{i j} x_{j}=\sum_{\substack{j=1 \\ j \neq i}}^{n} a_{i j} x_{j}=1 .
$$

According to Theorem 2, $S$ is an externally independent set of $G$.

Now let us remove a vertex $x_{i}$ from $S$ and denote the resulting vertex subset as $S^{\prime}$. The characteristic logical vector of $S^{\prime}$ is $V_{S^{\prime}}=\left[x_{1}, \ldots, x_{i-1}, 0, x_{i+1}, \ldots, x_{n}\right]$. Since $v_{i} \in S$, $x_{i}=1$. It follows from (36) that

$$
\sum_{\substack{j=1 \\ j \neq i}}^{n} a_{i j} x_{j}=0 .
$$

We then know that $\operatorname{col}_{j}(M)=0_{n}$, and this indicates that $V_{S^{\prime}}=\left[x_{1}, \ldots, x_{i-1}, 0, x_{i+1}, \ldots, x_{n}\right]$ does not meet (14). Therefore, $S^{\prime}$ is not an externally independent set of $G$. Thus, $S$ is a minimum externally independent set of $G$. The sufficiency is proved.

We can also transfer the problem of finding minimum externally independent sets of graphs to the problem of solving the minimum value of pseudo-Boolean functions. To this end, we first make some preparations. 
Lemma 1 (see [28]). Let $f\left(x_{1}, x_{2}, \ldots, x_{n}\right)$ be a pseudoBoolean function with constraint conditions:

$$
f_{j}\left(x_{1}, x_{2}, \ldots, x_{n}\right)=0, \quad j=1,2, \ldots, m,
$$

where each $f_{j}$ is a nonnegative integer-valued pseudoBoolean function. Denote by $S^{+}$and $S^{-}$the sums of positive coefficients and negative coefficients of $f$, respectively. Construct a pseudo-Boolean function:

$$
\begin{aligned}
F\left(x_{1}, x_{2}, \ldots, x_{n}\right)= & f\left(x_{1}, x_{2}, \ldots, x_{n}\right) \\
& +\left(S^{+}-S^{-}+1\right) \sum_{j=1}^{m} f_{j}\left(x_{1}, x_{2}, \ldots, x_{n}\right) .
\end{aligned}
$$

Then,

(i) If $\left(x_{1}^{*}, x_{2}^{*}, \ldots, x_{n}^{*}\right)$ is a local minimum point of $f$ under the constraint conditions (42), then $\left(x_{1}^{*}, x_{2}^{*}, \ldots, x_{n}^{*}\right)$ is also a local minimum point of $F$;

(ii) If $\left(x_{1}^{*}, x_{2}^{*}, \ldots, x_{n}^{*}\right)$ is a local minimum point of Fand $F\left(x_{1}^{*}, x_{2}^{*}, \ldots, x_{n}^{*}\right) \leq S^{+}$, then $\left(x_{1}^{*}, x_{2}^{*}, \ldots, x_{n}^{*}\right)$ is also a local minimum point of $f$ under the constraint conditions (42).

Theorem 4. For a given graph $G=(V, E)$ with the UAM $A=\left[a_{i j}\right]$, a vertex subset $S \subseteq V$ whose characteristic logical vector and characteristic value are $V_{S}=\left[x_{1}, x_{2}, \ldots, x_{n}\right]$ and $Y_{S}=\ltimes_{i=1}^{n} y_{i}=\delta_{2^{n}}^{k}$, respectively, where $y_{i}=\left[x_{i}, 1-x_{i}\right]^{T}$. Then, $S$ is a minimum externally independent set of $G$ if and only if the $k$-th component of $M$ is minimum among all the nonnegative components, where

$$
M=J\left(\sum_{i=1}^{n} Q_{i}+(n+1) \sum_{j=1}^{n} \sum_{i=1}^{n}\left(a_{i j} Q_{i}\right)\right),
$$

in which
$\left(E_{d_{-} \text {latter }}\right)^{i-1}$.

Proof. According to the proof of Theorem 1, the characteristic logical vector of a minimum externally independent set of graph $G$ is the local minimum point of pseudo-Boolean function $h\left(x_{1}, x_{2}, \ldots, x_{n}\right)=\sum_{i=1}^{n} x_{i}$ under the constraint condition (18). Using Lemma 1 , where $S^{+}=n$ and $S^{-}=0$, such a minimization problem with constraint conditions is converted to the following minimization problem without constraint conditions:

$$
f\left(x_{1}, x_{2}, \ldots, x_{n}\right)=\left(\bigvee_{i=1}^{n} x_{i}\right) \vee(n+1)\left(\bigvee_{i=1}^{n} \bigvee_{i=1}^{n}\left(a_{i j} x_{j}\right)\right) .
$$

Since both $x_{i}(i=1,2, \ldots, n)$ and $a_{i j}$ are two-valued variables taking 0 and $1,(45)$ is equivalent to the following pseudo-Boolean function:

$$
f\left(x_{1}, x_{2}, \ldots, x_{n}\right)=\sum_{i=1}^{n} x_{i}+(n+1) \sum_{j=1}^{n} \sum_{i=1}^{n}\left(a_{i j} x_{j}\right) .
$$

By (20)-(22), (46) can be rewritten as

$$
\begin{aligned}
f\left(y_{1}, y_{2}, \ldots, y_{n}\right)= & J\left(\sum_{i=1}^{n} Q_{i}\right) Y_{S} \\
& +(n+1)\left(\sum_{j=1}^{n} \sum_{i=1}^{n}\left(a_{i j} Q_{i}\right)\right) Y_{S} \\
= & J\left(\sum_{i=1}^{n} Q_{i}+(n+1) \sum_{j=1}^{n} \sum_{i=1}^{n}\left(a_{i j} Q_{i}\right)\right) \\
Y_{S}: & =M Y_{S},
\end{aligned}
$$

where

$$
\begin{aligned}
M & =J\left(\sum_{i=1}^{n} Q_{i}+(n+1) \sum_{j=1}^{n} \sum_{i=1}^{n}\left(a_{i j} Q_{i}\right)\right), \\
J & =[1,0], \\
Q_{i} & =\left(E_{d \_l a t t e r}\right)^{n-i} \ltimes W_{\left[2,2^{n-i}\right]} \ltimes\left(E_{d_{-} \text {latter }}\right)^{i-1}, y_{i} . \\
y_{i} & =\left[x_{i}, 1-x_{i}\right]^{T}, \\
Y_{S} & =\sum_{i=1}^{n}
\end{aligned}
$$

It is now clear from the characteristic value of $S$, $Y_{S}=\ltimes_{i=1}^{n} y_{i}=\delta_{2^{n}}^{k}$, that the $k$-th component of $M$ is the minimum value of functions (45) and (46). The proof is completed.

Remark 6. The necessity and sufficiency of Theorem 4 ensure that the matrix of (44) is qualified to describe the minimum externally independent sets of graphs. We define $M$ as the minimum externally independent set matrix of a graph.

Definition 10 (minimum externally independent set matrix). Matrix $M$ of (44) in Theorem 4 is called the minimum externally independent set matrix of graph $G$.

The necessity and sufficiency of Theorem 4 also offer us a matrix approach to find all the minimum externally independent sets of a graph. Similar to Algorithm 1, we have the following algorithm producing all the minimum externally independent sets of a given graph.

Algorithm 2. Let $A=\left[a_{i j}\right]$ be the UAM of graph $G=(V, E)$. Assign each vertex $v_{i} \in V$ a characteristic variable $x_{i} \in D=$ $\{0,1\}$ and define $y_{i}=\left[x_{i}, 1-x_{i}\right]^{T}$. Taking the following steps, one can get all the minimum externally independent sets of $G$. 
Step 1. Compute the minimum externally independent set matrix of $G$, that is, $M$ in (44).

Step 2. Check whether there are nonnegative components in the first row of $M$; if not, $G$ contains no minimum externally independent set and the algorithm terminates. Otherwise, set

$$
K=\left\{l \mid \operatorname{col}_{l}(M)=\min (\operatorname{col}(M))\right\} .
$$

Step 3. For each $l$ in $K$, solve the equation with respect to $y_{i}, \quad \ltimes_{i=1}^{n} y_{i}=\delta_{2^{n}}^{l}, \quad$ by computing $y_{i}=S_{i}^{n} \ltimes \delta_{2^{n}}^{l}$, $i=1,2, \ldots, n$, where

$$
\left\{\begin{array}{l}
S_{1}^{n}=\delta_{2}[\underbrace{1, \ldots, 1}_{2^{n-1}} \underbrace{2, \ldots, 2}_{2^{n-1}}], \\
S_{2}^{n}=\delta_{2}[\underbrace{1, \ldots, 1}_{2^{n-2}} \underbrace{2, \ldots, 2}_{2^{n-2}} \underbrace{1, \ldots, 1}_{2^{n-2}} \underbrace{2, \ldots, 2}_{2^{n-2}}], \\
S_{n}^{n}=\delta_{2} \underbrace{[\underbrace{12}_{2}, \ldots, \underbrace{12}_{2}]}_{2^{n-1}} .
\end{array}\right.
$$

Step 4. Choose $y_{i}=\delta_{2}^{1}$ and set

$$
S_{l}=\left\{v_{i} \mid y_{i}=\delta_{2}^{1}\right\}
$$

$S_{l}$ is a minimum externally independent set of $G$; and all the minimum externally independent sets of $G$ are $\left\{S_{l} \mid l \in K, S_{l}\right.$ is produced by Steps 3 and 4.$\}$

3.3. Extending Results to the Kernels of Graphs. The kernel of graphs is an important topic in the area of graph theory and has a great applied value in the scientific field such as similarity measurement of graph structures [3]. A subset $S$ of vertices is called a kernel of a graph if $S$ is both an externally independent set and an internally stable set of the graph. The matrix formulation and algebraic algorithm of internally stable set of graphs are presented in [26]. Combining these results, in this subsection, we extend the results of the externally independent sets and minimum externally independent sets to the case of the kernels of graphs, including matrix formulation and algebraic algorithm of finding all the kernels of a graph.

Lemma 2 (see [26]). Let $A=\left[a_{i j}\right]$ be the UAM of graph $G=$ $(V, E)$ with $V=\left\{v_{1}, v_{2}, \ldots, v_{n}\right\}$. Then, $G$ has an internally stable set if and only if there is $j, 1 \leq j \leq 2^{n}$, such that $\operatorname{col}_{j}(N)=0_{n}$, where

$$
\begin{aligned}
N & =\left[\begin{array}{c}
N_{1} \\
N_{2} \\
\vdots \\
N_{n}
\end{array}\right], \\
N_{i} & =Q \sum_{j=1}^{n} a_{i j} P_{i j}, \quad i=1,2, \ldots, n, \\
Q & =[1,0,0,0], \\
P_{i j} & =\left(E_{d_{-} \text {latter }}\right)^{n-2} \ltimes W_{\left[2^{j}, 2^{n-j}\right]} \ltimes W_{\left[2^{i}, 2^{j-i-1}\right]} .
\end{aligned}
$$

Lemma 3 (see [26]). Let $A=\left[a_{i j}\right]$ be the UAM of graph $G=$ $(V, E)$ with $V=\left\{v_{1}, v_{2}, \ldots, v_{n}\right\}$. For a given subset $S \subseteq V$ with characteristic logical vector $V_{S}=\left[x_{1}, x_{2}, \cdots, x_{n}\right]$ and letting $Y_{S}=\ltimes_{i=1}^{n} y_{i}=\delta_{2^{n}}^{k}, y_{i}=\left[x_{i}, \bar{x}_{i}\right]^{T}, S$ is an internally stable set of $G$ if and only if $\operatorname{col}_{k}(N)=0_{n}$, where $N$ is given in Lemma 2.

According to the definition of kernel of a graph, combining Lemmas 2 and 3 and Theorems 1 and 2, we can easily get the following results.

Corollary 2. Given a graph $G=(V, E)$ with $A=\left[a_{i j}\right]$ being its UAM, $V=\left\{v_{1}, v_{2}, \ldots, v_{n}\right\}$. G contains a kernel if and only if there is $1 \leq j \leq 2^{n}$ satisfying the conditions:

$$
\left\{\begin{array}{l}
\operatorname{col}_{j}(M)=1_{n}, \\
\operatorname{col}_{j}(N)=0_{n},
\end{array}\right.
$$

where $M$ and $N$ are given in Theorem 1 and Lemma 2, respectively.

Corollary 3. Let $A=\left[a_{i j}\right]$ be the UAM of graph $G=(V, E)$. Given a vertex subset $S \subseteq V$ with $V_{S}=\left[x_{1}, x_{2}, \ldots, x_{n}\right]$ being its characteristic logical vector and $Y_{S}=\ltimes_{i=1}^{n} y_{i}=\delta_{2^{n}}^{k}$ being its characteristic value, where $y_{i}=\left[x_{i}, 1-x_{i}\right]^{T}, S$ is a kernel $G$ if and only if

$$
\left\{\begin{array}{l}
\operatorname{col}_{k}(M)=1_{n}, \\
\operatorname{col}_{k}(N)=0_{n},
\end{array}\right.
$$

where $M$ and $N$ are given in Corollary 2.

From Corollaries 2 and 3, it is clear that matrices $M$ and $N$ present the information of all the kernels of a graph. We introduce the definition of kernel matrix of a graph.

Definition 11 (kernel matrix of graphs). The following matrix $K$ is called the kernel matrix of graph $G$ described in Corollary 3, where

$$
K=\left(\begin{array}{l}
M \\
N
\end{array}\right) \text {, }
$$

in which $M$ and $N$ are given in Corollary 3.

Corollary 3 offers us a way to develop an algorithm to find all the kernels of a graph. 
Algorithm 3 Consider the graph given in Corollary 3. Assign each $v_{i} \in V$ a two-valued variable $x_{i} \in D=\{0,1\}$, and define $y_{i}=\left[x_{i}, 1-x_{i}\right]^{T}$. The following procedure produces all the kernels of $G$ :

Step 1: compute the kernel matrix of graph $G$, denoted as $K$.

Step 2: divide $K$ into the form of $K=\left(\begin{array}{c}M \\ N\end{array}\right)$ as de-
scribed in Corollary 3 . Step 3: check whether $M$ has a column being $1_{n}$. If not, $G$ has no kernel set and the algorithm ends here. Otherwise, go to next step.

Step 4: check whether $N$ has a column being $0_{n}$. If not, $G$ has no kernel set and the algorithm ends here. Otherwise, set

$$
R=\left\{l \mid \operatorname{col}_{l}(M)=1_{n}, \operatorname{col}_{l}(N)=0_{n}\right\}
$$

Step 5: solve the equation $\ltimes_{i=1}^{n} y_{i}=\delta_{2^{n}}^{l}$ with respect to $y_{i}$ for each $l$ in $R$ by computing $y_{i}=S_{i}^{n} \ltimes \delta_{2^{n}}^{l}, i=1,2, \ldots, n$, where

$$
\left\{\begin{array}{l}
S_{1}^{n}=\delta_{2}[\underbrace{1, \ldots, 1}_{2^{n-1}} \underbrace{2, \ldots, 2}_{2^{n-1}}], \\
S_{2}^{n}=\delta_{2}[\underbrace{1, \ldots, 1}_{2^{n-2}} \underbrace{2, \ldots, 2}_{2^{n-2}} \underbrace{1, \ldots, 1}_{2^{n-2}} \underbrace{2, \ldots, 2}_{2^{n-2}}], \\
S_{n}^{n}=\delta_{2} \underbrace{[\underbrace{12}_{2}, \ldots, \underbrace{12}_{2}]}_{2^{n-1}} .
\end{array}\right.
$$

Step 6: select $y_{i}=\delta_{2}^{1}$ and construct $S_{l}=\left\{v_{i} \mid y_{i}=\delta_{2}^{1}\right\} . S_{l}$ is a kernel of $G$. All the kernels of $G$ are $\left\{S_{l} \mid l \in K\right\}$.

\section{Comparisons with Existing Approaches and Complexity Analysis}

The externally independent set problem of graphs has been studied widely and many results have been proposed. Most of the existing results can be roughly classified, from viewpoint of research approach, into three categories: graph theory-based, integer programming model-based, and algorithm-based [29].

The STP of matrices provides an elegant way to analyze the structures of graphs [20], which is very promising due to the rapid increase of computational technologies. The results of this paper established using the STP of matrices have the following differences and advantages in the aspects of model formulation, perspective and expansibility of conclusions, and so forth.

Most of existing results use graph theory, optimization theory, and computer-based algorithms to formulate and find externally independent sets of a graph. Such approach has a disadvantage that not all the externally independent sets of given graphs can be obtained. In this paper, the problem of externally independent set is considered from the perspective of $\mathrm{k}$-valued logic. Using the algebraic expression of k-valued pseudo-Boolean functions developed by STP, the externally independent set matrix (EISM), minimum externally independent set matrix (MEISM), and kernel matrix are proposed. These three matrices have precise mathematical meanings; they provide complete information of externally independent sets (EISs), minimum externally independent sets (MEISs), and kernel sets of a graph. More importantly, the completeness of describing externally independent sets (EISs) and minimum externally independent sets (MEISs) and kernels of graphs enable us to design algorithms to find all the EISs, MEISs, and kernel sets of a graph.

Besides, the proposed results, including theoretical results and algorithms, can be easily extended to the cases of $\mathrm{k}$-externally independent set, $\mathrm{k}$-minimum externally independent set, and k-kernel sets by replacing the unitary adjacency matrix (UAM) by k-adjacency matrix (KAM) of graphs. Correspondingly, the concepts of k-externally independent set matrix (k-EISM), minimum externally independent set matrix (k-MEISM), and k-kernel matrix can also be established.

We next analyze the computational complexity of the presented searching algorithms (Algorithms 1-3), taking Algorithm 2 as an example; Algorithms 1 and 3 are very similar to Algorithm 2. Consider a graph $G$ of $n$ vertices $V=\left\{v_{1}, v_{2}, \ldots, v_{n}\right\}$. In Algorithm 2, only Step 3 involves circulation iteration. For a graph with $n$ vertices, the time of circulation iteration is at most $n-1$ in the worst situation. Therefore, Algorithm 2 iterates $n-1$ times at most and the time complexity is $O(n)$. In addition, all the computations involved in Algorithm 2 are matrix multiplication, namely, the semitensor product of matrices, which can be easily conducted by using the STP toolbox.

\section{An Application to WSN Covering Problems}

WSN covering problem studies how to reach a minimum number of sensors or the construction costs under the condition of covering all points of interest. WSN covering problem can be studied by viewing it as set covering problem. The set covering problem was first proposed by Roth [30] to solve the location problem of emergency service facilities such as fire control center and ambulance. An integer programming model for the set covering problem with different costs was established. Plane, Hendrick, Daskin, and Stern proposed a double objective set covering problem with the minimum number of service stations and the maximum number of customers. Since 2000, many heuristic algorithms have been used to solve set covering problems. Fisher and Northup proposed a dual heuristic algorithm to solve the set covering problem [31]. Beasley and Jornsten combined the subgradient optimization method and Lagrange relaxation algorithm to solve such problems [32]. Beasley and Chu gave a genetic algorithm to solve the set covering problem with different cost of service stations. 
Among them, random greedy algorithm, simple greedy algorithm, and transform greedy algorithm are the best algorithms for almost all problems, of which random greedy algorithm is the best. Halperin and Karp proved that the set covering problem is NP-complete [33].

In this section, we use the presented results to solve a WSN covering problem, whose graph model is shown in Figure 1, to verify the correctness and effectiveness of the results. It is sufficient from the logical relation among Algorithm 2, Theorems 2 and 1, and Algorithm 1 that there is only a need to check Algorithm 2.

The UAM of $G$ is

$$
A=\left[\begin{array}{lllllllll}
0 & 1 & 0 & 0 & 0 & 0 & 0 & 1 & 0 \\
1 & 0 & 1 & 1 & 1 & 0 & 0 & 1 & 0 \\
0 & 1 & 0 & 1 & 0 & 0 & 0 & 0 & 0 \\
0 & 1 & 1 & 0 & 1 & 1 & 1 & 1 & 1 \\
0 & 1 & 0 & 1 & 0 & 1 & 0 & 0 & 0 \\
0 & 0 & 0 & 1 & 1 & 0 & 1 & 0 & 0 \\
0 & 0 & 0 & 1 & 0 & 1 & 0 & 0 & 0 \\
1 & 1 & 0 & 1 & 0 & 0 & 0 & 0 & 0 \\
0 & 0 & 0 & 1 & 0 & 0 & 0 & 0 & 0
\end{array}\right] .
$$

Steps 1 and 2: according to (44), all of the minimum nonnegative components of the minimum externally independent set matrix of $G$ are as follows:

$$
\begin{array}{r}
\operatorname{col}_{171}(M), \operatorname{col}_{183}(M), \operatorname{col}_{224}(M), \\
\operatorname{col}_{352}(M), \operatorname{col}_{374}(M), \operatorname{col}_{375}(M), \\
\operatorname{col}_{378}(M), \operatorname{col}_{379}(M), \operatorname{col}_{426}(M), \\
\operatorname{col}_{438}(M), \operatorname{col}_{478}(M) .
\end{array}
$$

Thus, the set $K$ is $K=\{171,183,224,352,374,375$, $378,379,426,438,478\}$.

Step 3: for each $l$ in $K$, computing $y_{i}=S_{i}^{9} \ltimes \delta_{512}^{l}$, $i=1,2, \ldots, 9$, taking $l=171$ as an example, we get

$$
\begin{aligned}
& y_{1}=S_{1}^{9} \ltimes \delta_{512}^{171}=\delta_{2}^{1}, \\
& y_{2}=S_{2}^{9} \ltimes \delta_{512}^{171}=\delta_{2}^{2}, \\
& y_{3}=S_{3}^{9} \ltimes \delta_{512}^{171}=\delta_{2}^{1}, \\
& y_{4}=S_{4}^{9} \ltimes \delta_{512}^{171}=\delta_{2}^{2}, \\
& y_{5}=S_{5}^{9} \ltimes \delta_{512}^{171}=\delta_{2}^{1}, \\
& y_{6}=S_{6}^{9} \ltimes \delta_{512}^{171}=\delta_{2}^{2}, \\
& y_{7}=S_{7}^{9} \ltimes \delta_{512}^{171}=\delta_{2}^{1}, \\
& y_{8}=S_{8}^{9} \ltimes \delta_{512}^{171}=\delta_{2}^{2}, \\
& y_{9}=S_{9}^{9} \ltimes \delta_{512}^{171}=\delta_{2}^{1} .
\end{aligned}
$$

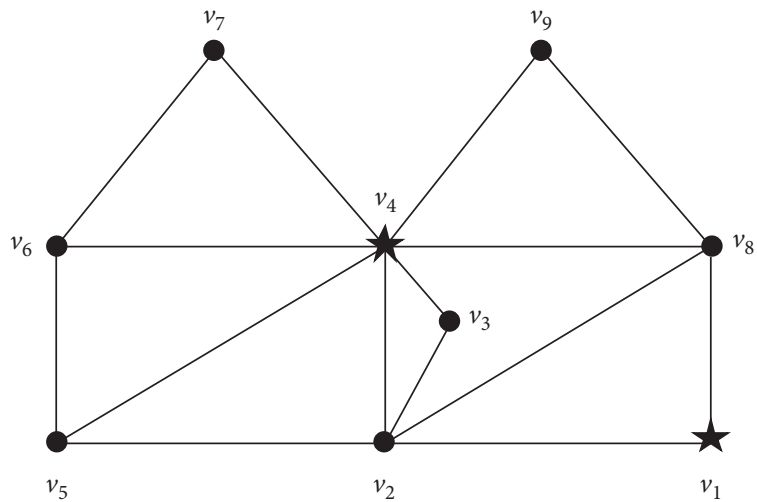

Figure 1: Graph model of a WSN covering problem.

TABLE 1: All the minimum externally independent sets (MEISs) of G.

\begin{tabular}{lc}
\hline$l$ & MEIS \\
\hline 171 & $\left\{v_{1}, v_{3}, v_{5}, v_{7}, v_{9}\right\}$ \\
183 & $\left\{v_{1}, v_{3}, v_{6}, v_{9}\right\}$ \\
224 & $\left\{v_{1}, v_{4}\right\}$ \\
352 & $\left\{v_{2}, v_{4}\right\}$ \\
374 & $\left\{v_{2}, v_{6}, v_{8}\right\}$ \\
375 & $\left\{v_{2}, v_{6}, v_{9}\right\}$ \\
378 & $\left\{v_{2}, v_{7}, v_{8}\right\}$ \\
379 & $\left\{v_{2}, v_{7}, v_{9}\right\}$ \\
426 & $\left\{v_{3}, v_{5}, v_{7}, v_{8}\right\}$ \\
438 & $\left\{v_{3}, v_{6}, v_{8}\right\}$ \\
478 & $\left\{v_{4}, v_{8}\right\}$ \\
\hline
\end{tabular}

Step 4: choose $y_{i}=\delta_{2}^{1}$ and construct $S_{171}=\left\{v_{i} \mid y_{i}=\right.$ $\left.\delta_{2}^{1}\right\}=\left\{v_{1}, v_{3}, v_{5}, v_{7}, v_{9}\right\} . S_{171}$ is a minimum externally independent set of $G$.

Repeating Steps 3 and 4, we get all the minimum externally independent sets of $G$, which are listed in Table 1.

It is clear from Table 1 that $G$ has three absolutely minimum externally independent sets:

$$
\begin{aligned}
& \left\{v_{1}, v_{4}\right\}, \\
& \left\{v_{2}, v_{4}\right\}, \\
& \left\{v_{4}, v_{8}\right\} .
\end{aligned}
$$

Therefore, the covering problem has three optimal solutions:

(1) Putting two sensors at locations $v_{1}$ and $v_{4}$

(2) Putting two sensors at locations $v_{2}$ and $v_{4}$

(3) Putting two sensors at locations $v_{4}$ and $v_{8}$

Remark 7. It was shown in [28] that there are two optimization schemes of putting two sensors, $\left\{v_{1}, v_{4}\right\}$ and $\left\{v_{2}, v_{4}\right\}$. According to our result, there are exactly three such optimization schemes, $\left\{v_{1}, v_{4}\right\},\left\{v_{2}, v_{4}\right\}$, and $\left\{v_{4}, v_{8}\right\}$. 


\section{Concluding Remarks}

Externally independent set is an important structure of a graph. Many problems in science and engineering can be formulated by externally independent set of graphs. Description and search of externally independent sets of a graph represent a key issue in graph theory. In this paper, a newly proposed matrix analysis tool, semitensor product of matrices, is used to consider the problem of formulating and finding externally independent sets, minimum externally independent sets, and kernel sets of graphs. Several new necessary and sufficient conditions of these three structures are obtained; and three kinds of matrices containing the complete information of these vertex subsets are proposed. Further three algebraic algorithms that can find all these special subsets of a graph are developed. As a test of the effectiveness of the obtained results, a WSN covering problem, namely, location of service stations, is considered by these results. The research approach of this paper may offer a different way to investigate the structures of graphs and related problems such as Hamiltonian paths, Hamiltonian circles, and complete graphs.

\section{Notations}

$$
\begin{array}{ll}
1_{n} / 0_{n}: & \text { The } n \times 1 \text { vector with each element being } 1 / 0 \\
\operatorname{col}_{\mathrm{i}}(A): & \text { The } i \text {-th column of matrix } A \\
\delta_{n}^{i}: & \text { The } i \text {-th column of the identity matrix } \\
& I_{n} \in \mathbf{M}_{n \times n} \\
\Delta_{n}: & \text { A set }\left\{\delta_{n}^{1}, \ldots, \delta_{n}^{n}\right\} \\
\delta_{n}\left[i_{1}, \ldots, i_{k}\right]: & \text { The compactness form of matrix }\left[\delta_{n}^{i_{1}}, \ldots, \delta_{n}^{i_{k}}\right] \\
|S|: & \text { The cardinality of set } S .
\end{array}
$$

\section{Data Availability}

No underlying data support the results of the current study.

\section{Disclosure}

The funders had no role in the design of the study; in the collection, analyses, or interpretation of data; in the writing of the manuscript; or in the decision to publish the results.

\section{Conflicts of Interest}

The authors declare that there are no conflicts of interest.

\section{Acknowledgments}

The authors would like to express their thanks to Professor Yiguang Hong for his encouragement and helpful suggestions. This research was funded by the National Natural Science Foundation of China (grant nos. U1804150 and 62073124).

\section{References}

[1] J. Richard, Introduction to Graph Theory, PMA Publishing, London, UK, 2017.

[2] X. L. Li, C. Magnant, and Z. M. Qin, "Properly colored connectivity of graphs introduction," 2018.
[3] G. Lin and J. Guan, "Solving maximum set k-covering problem by an adaptive binary particle swarm optimization method," Knowledge-based Systems, vol. 142, pp. 95-107, 2018.

[4] Y. Y. Wang, D. T. Ouyang, L. M. Zhang et al., "A novel local search for unicost set covering problem using hyperedge configuration checking and weight diversity," Science ChinaInformation Sciences, vol. 60, no. 6, 2017.

[5] J. M. Yue and Y. Y. Yan, "Matrix method to search k-maximum internally stable sets of graphs," Proceedings of the 34th Chinese Control Conference, vol. 23, pp. 36-41, 2015.

[6] H. Hua, "A sharp upper bound for the number of stable sets in graphs with given number of cut edges," Applied Mathematics Letters, vol. 22, no. 9, pp. 1380-1385, 2009.

[7] X. Deng, G. Li, and W. Zang, "Corrigendum to Proof of Chvátal's conjecture on maximal stable sets and maximal cliques in graphs," Journal of Combinatorial Theory, Series B, vol. 94, no. 2, pp. 352-353, 2005.

[8] G. L. Ding, "Stable sets versus independent sets," Discrete Mathematics, vol. 117, no. 1-3, pp. 73-87, 2005.

[9] J. Sun and Z.-Q. Hu, "Proof of Ding's conjecture on maximal stable sets and maximal cliques in planar graphs," Acta Mathematicae Applicatae Sinica, English Series, vol. 26, no. 3, pp. 473-480, 2010.

[10] D. Z. Cheng, H. S. Qi, and Y. Zhao, An Introduction to Semi-tensor Product of Matrices and its Applications, World Scientific Publishing Co. Pte. Ltd., Singapore, 2012.

[11] S. Zhu, J. Lu, Y. Lou, and Y. Liu, "Induced-equations-based stability analysis and stabilization of markovian jump boolean networks," IEEE Transactions On Automatic Control, vol. 1, 2020.

[12] J. Q. Lu, B. W. Li, and J. Zhong, "A novel synthesis method for reliable feedback shift registers via Boolean networks," Science China: Information Sciences, vol. 64, no. 5, p. 152207, 2021.

[13] Y. Yang, Y. Liu, Y. Liu, J. Lou, and Z. Wang, "Observability of switched Boolean control networks using algebraic forms," Discrete \& Continuous Dynamical Systems-S, vol. 14, no. 4, pp. 1519-1533, 2021.

[14] S. Le, Y. Wu, and M. Toyoda, "A congestion game framework for service chain composition in NFV with function benefit," Information Sciences, vol. 514, pp. 512-522, 2020.

[15] J. T. Zhao, Z. Q. Chen, and Z. X. Liu, "A novel matrix approach for the stability and stabilization analysis of colored Petri nets," Science China-Information Sciences, vol. 62, no. 9, 2019.

[16] Q. Zhu, Z. Gao, Y. Liu, and W. Gui, "Categorization problem on controllability of boolean control networks," IEEE Transactions On Automatic Control, vol. 1, 2020.

[17] Z. Chen, Y. Zhou, Z. Zhang, and Z. Liu, "Semi-tensor product of matrices approach to the problem of fault detection for discrete event systems (DESs)," IEEE Transactions on Circuits and Systems II: Express Briefs, vol. 67, no. 12, pp. 3098-3102, 2020.

[18] Z. P. Zhang, C. Y. Xia, and Z. Q. Chen, "On the stabilization of nondeterministic finite automata via static output feedback [J]," Applied Mathematics And Computation, vol. 365, pp. 872-880, 2020.

[19] Y. R. Zhou, Z. Q. Chen, Z. X. Liu et al., Strongly Fault Prognosability of Partially-Observed Discrete Event Systems, CIENCE CHINA-Information Sciences, London, UK, 2021.

[20] Y. Wang, C. Zhang, and Z. Liu, "A matrix approach to graph maximum stable set and coloring problems with application to multi-agent systems," Automatica, vol. 48, no. 7, pp. 1227-1236, 2012. 
[21] M. Xu, Y. Wang, and A. Wei, "Robust graph coloring based on the matrix semi-tensor product with application to examination timetabling," Control Theory and Technology, vol. 12, no. 2, pp. 187-197, 2014.

[22] M. R. Xu and Y. Z. Wang, "T-coloring of graphs with application to frequency assignment in cellular mobile networks," Proceedings of the 33rd Chinese Control Conference, vol. 23, pp. 2536-2541, 2014.

[23] M. R. Xu, Y. Z. Wang, and P. Jiang, "Fuzzy graph coloring via semi-tensor product method," Proceedings of the 34th Chinese Control Conference, vol. 23, pp. 973-978, 2015.

[24] J. Zhong, J. Lu, C. Huang, L. Li, and J. Cao, "Finding graph minimum stable set and core via semi-tensor product approach," Neurocomputing, vol. 174, pp. 588-596, 2016.

[25] M. Meng, J. E. Feng, and X. X. Li, "A matrix method to hypergraph transversal and covering problems with application in simplifying Boolean functions," Proceedings of the 35th Chinese Control Conference, vol. 2016, pp. 2772-2777, 2016.

[26] J. M. Yue, Z. Q. Chen, Y. Y. Yan et al., "Solvability of k-track assignment problem: a graph approach," Control Theory \& Applications, vol. 34, no. 4, pp. 457-466, 2017.

[27] Y. Yan, J. Yue, Z. Chen, and Y. Liu, "Algebraic expression and construction of control sets of graphs using semi-tensor product of matrices," IEEE Access, vol. 7, pp. 113440-113451, 2019.

[28] Y. C. Liu and W. Zhang, Boolean Methodology, Literature Press, London, UK, 1993.

[29] H. Hua and Y. Hou, "On graphs with the third largest number of maximal independent sets," Information Processing Letters, vol. 109, no. 4, pp. 248-253, 2009.

[30] J. P. Roth, "Algorithm for computing an almost minimum cover," IBM Technical Disclosure Bulletin, vol. 15, no. 12, pp. 3720-3722, 1973.

[31] M. L. Fisher, W. D. Northup, and J. F. Shapiro, "Using duality to solve discrete optimization problems: theory and computational experience," Nondifferentiable Optimization, vol. 4, pp. 56-94, 1975.

[32] J. E. Beasley and K. Jørnsten, "Enhancing an algorithm for set covering problems," European Journal of Operational Research, vol. 58, no. 2, pp. 293-300, 1992.

[33] E. Halperin and R. M. Karp, "The minimum-entropy set cover problem," 2004. 